\title{
3 Research Square

\section{Searching for refractory plasmonic materials: the structural and optical properties of Au3Zr intermetallic thin films}

Hugh Littlehailes ( $\sim$ h.littlehailes.1@research.gla.ac.uk)

Queen's University Belfast School of Mathematics and Physics https://orcid.org/0000-0003-4815-7878 William R Hendren

Queen's University Belfast School of Mathematics and Physics

Stacey Drakeley

Queen's University Belfast School of Mathematics and Physics

Robert M Bowman

Queen's University Belfast School of Mathematics and Physics

Fumin Huang

Queen's University Belfast School of Mathematics and Physics

\section{Research Article}

Keywords: refractory plasmonic materials, Au3zr

Posted Date: March 22nd, 2021

DOI: https://doi.org/10.21203/rs.3.rs-296684/v1

License: (9) This work is licensed under a Creative Commons Attribution 4.0 International License. Read Full License 


\section{Abstract}

Optical properties of refractory intermetallic thin films of $\mathrm{Au} 3 \mathrm{Zr}$ were experimentally investigated for the first time, which show distinctive plasmonic properties in the visible and near infrared region. The films were fabricated through DC magnetron sputtering at various deposition temperature ranging from room temperature to $427^{\circ} \mathrm{C}$ and annealed at different vacuum levels. Both the structural and optical properties are found to be critically dependent on deposition temperature and anneal conditions. Films deposited between $205-320^{\circ} \mathrm{C}$ are shown to exhibit lower negative permittivity and better thermal stability, which could be linked to specific crystalline orientations. The films are stable when annealed at $10-8$ Torr, but are partially oxidized when annealed at $10-6$ Torr, suggesting oxidization could be a restricting issue for high-temperature applications in ambient environment.

\section{Full Text}

Due to technical limitations, full-text HTML conversion of this manuscript could not be completed. However, the latest manuscript can be downloaded and accessed as a PDF.

\section{Figures}




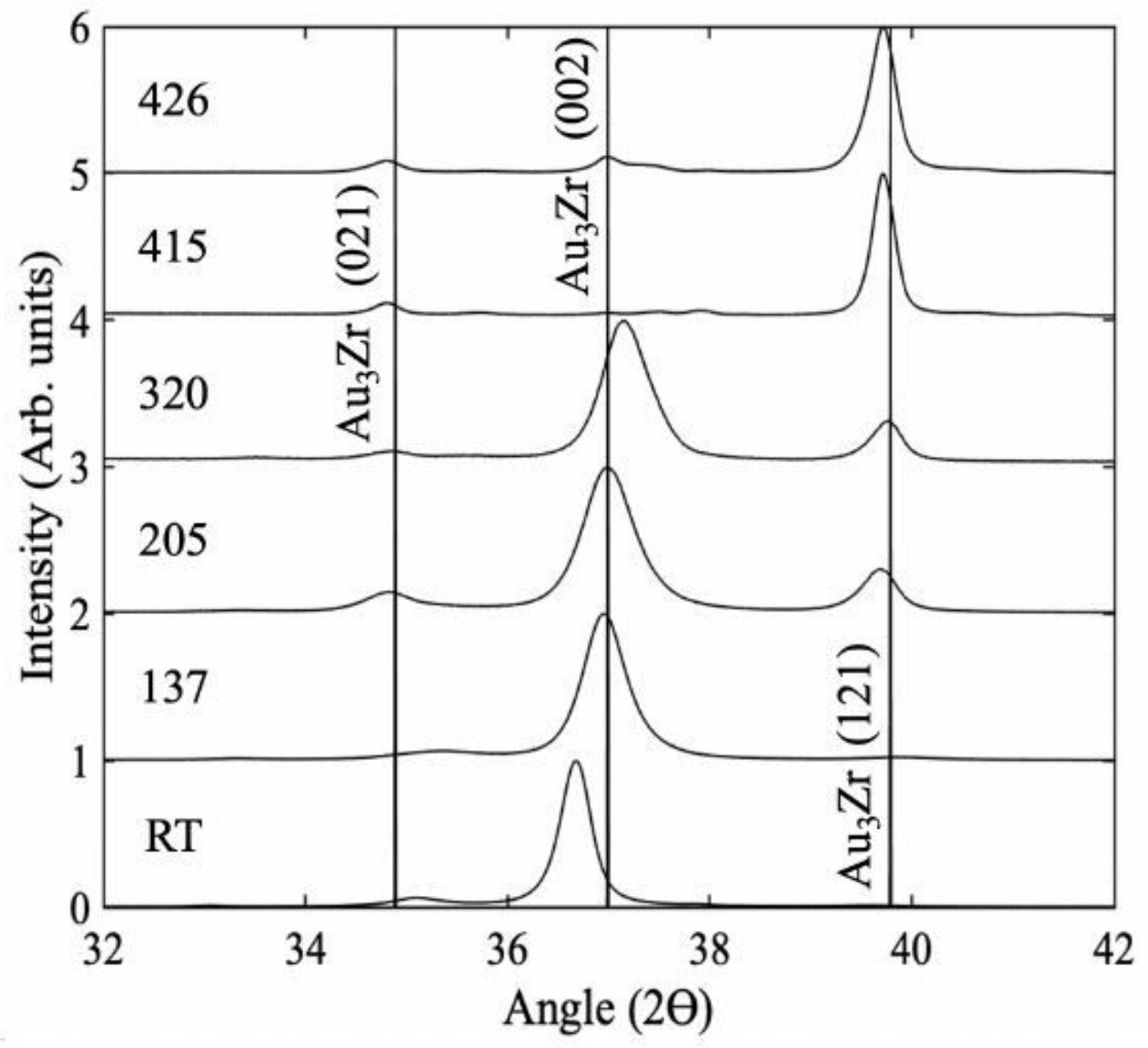

Figure 1

XRD spectra of Au3Zr films fabricated at different deposition temperatures denoted by the numbers (in the unit of Celsius). Solid lines indicate the expected peak positions calculated from the lattice parameters in literature 

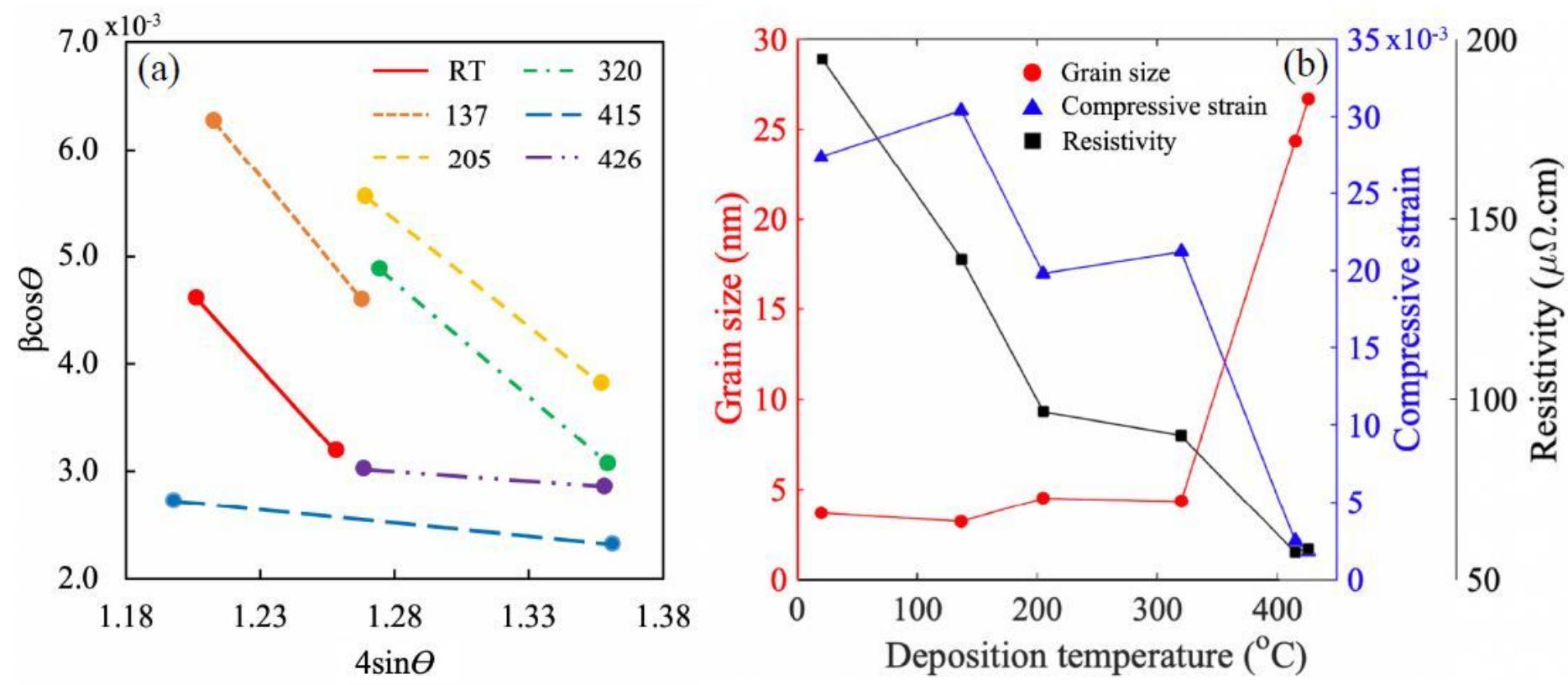

Figure 2

Williamson-Hall analysis of the XRD results. (a) Williamson-Hall plots of films deposited at various temperatures denoted by the numbers $\left({ }^{\circ} \mathrm{C}\right)$. (b) The average grain size (circles), strain (triangles) and resistivity (squares) of the Au3Zr films as a function of deposition temperature 

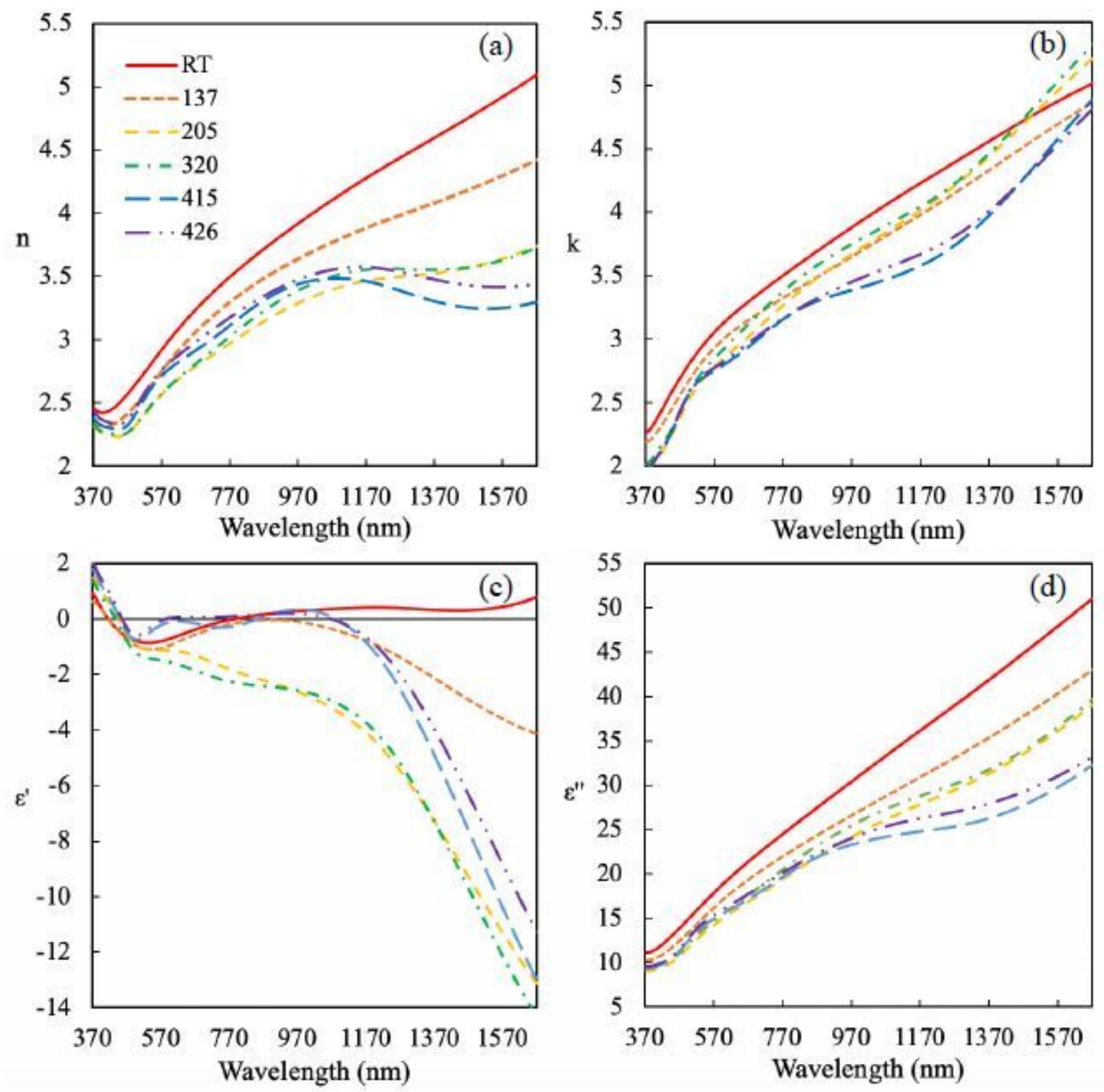

Figure 3

Optical properties of Au3Zr thin films deposited at various temperatures. (a-b) Real (n) and imaginary (k) part of refractive index. (c-d) Real $\left(\varepsilon^{\prime}\right)$ and imaginary $\left(\varepsilon^{\prime \prime}\right)$ part of permittivity 

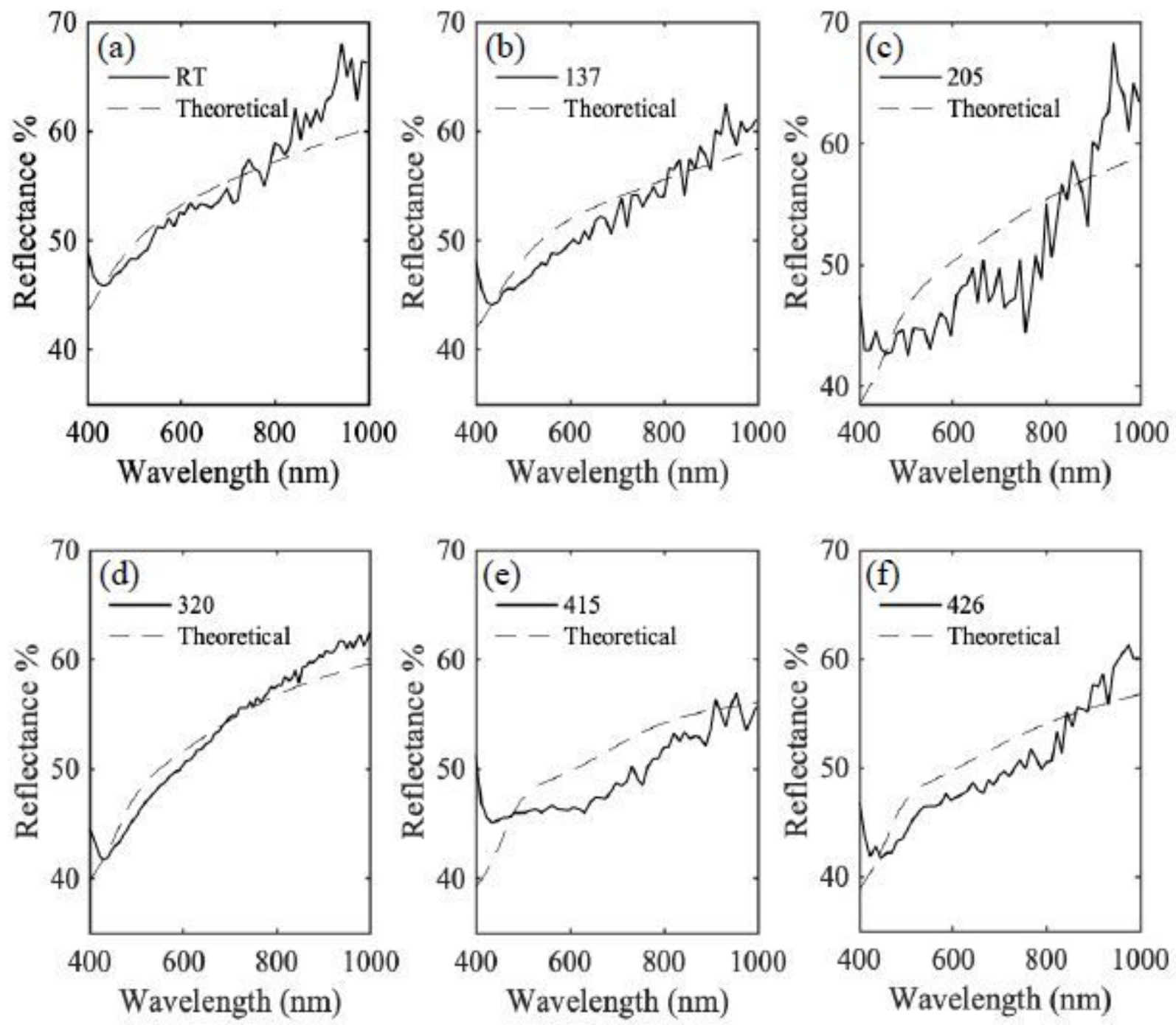

\section{Figure 4}

Measured (solid) and calculated (dashed) optical reflectance spectra of Au3Zr thin films fabricated at various deposition temperatures 

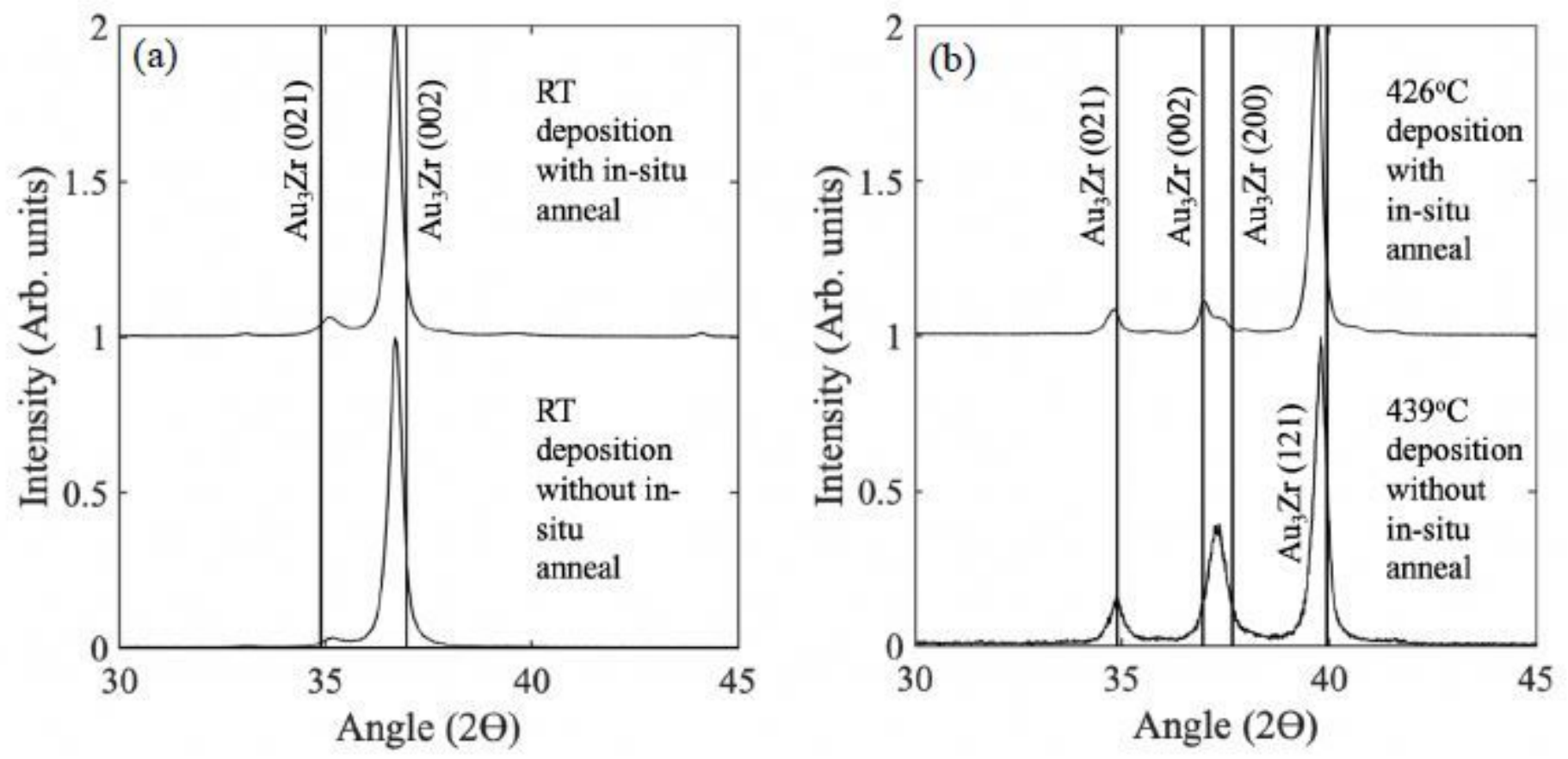

Figure 5

Effects of in-situ anneal. Comparison of XRD spectra of Au3Zr films deposited at (a) room temperature (RT) and (b) $426^{\circ} \mathrm{C}$ and $4390^{\circ}$, with and without in-situ anneal, respectively
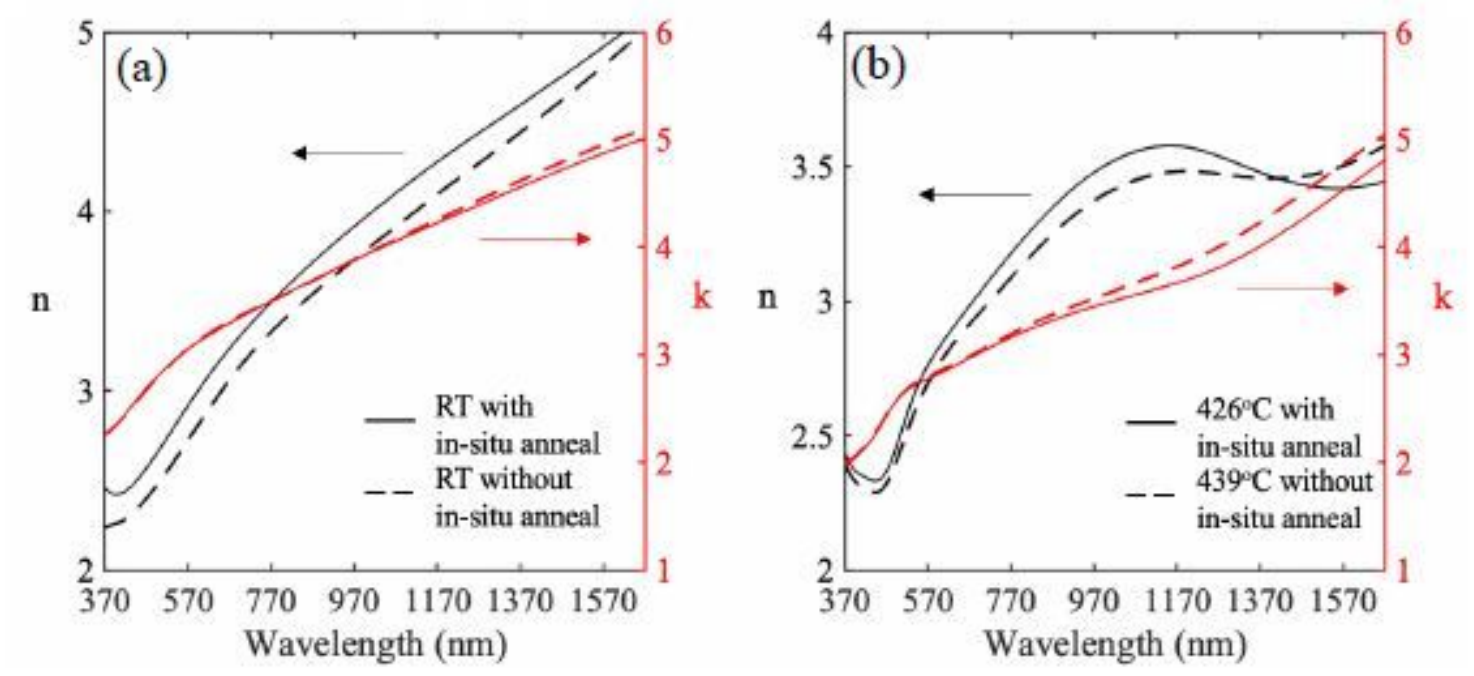

Figure 6

The effects of in-situ anneal on the optical properties. Real and imaginary refractive index of Au3Zr films with and with-out the in-situ anneal. (a) films deposited at RT, (b) films deposited at 426oC (in-situ annealed sample) and $4390 \mathrm{C}$ (without in-situ anneal) 


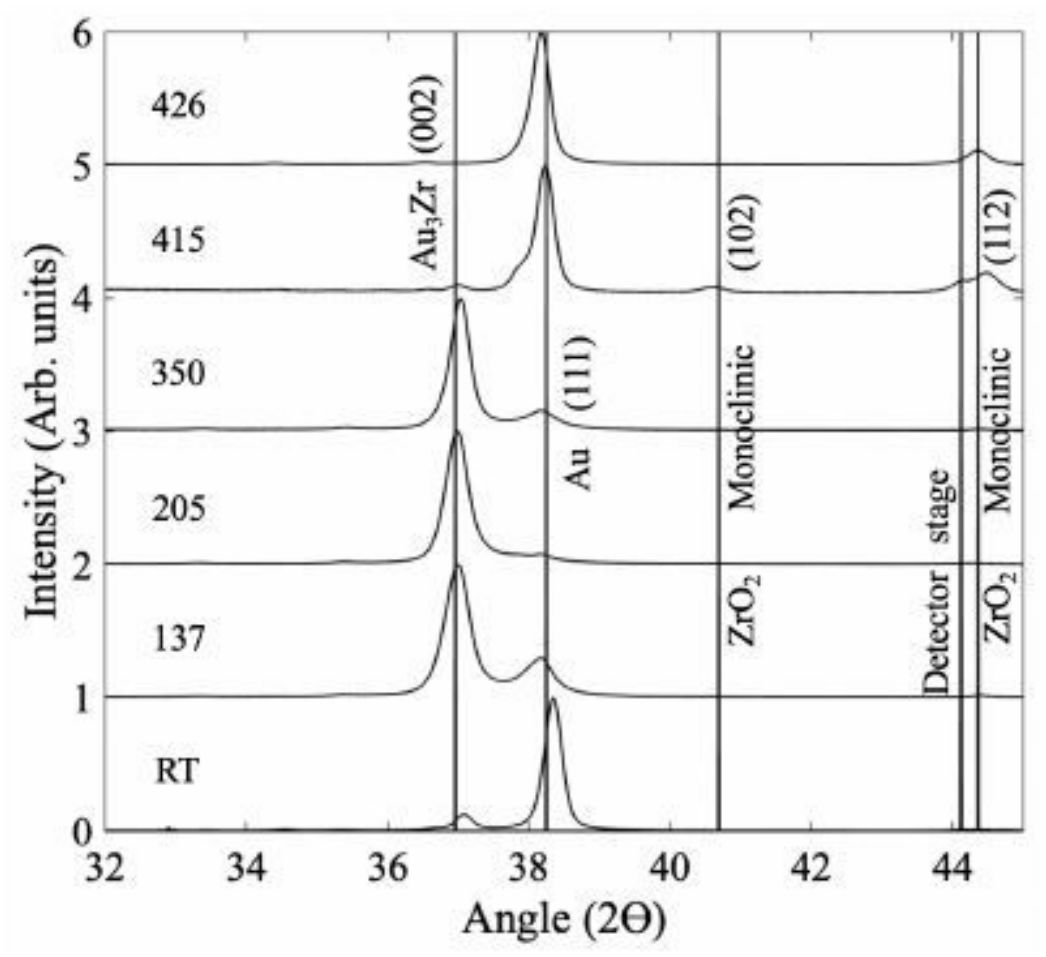

Figure 7

XRD spectra of Au3Zr films annealed in a vacuum oven (1.5×10-6 Torr) at $497^{\circ} \mathrm{C}$ for 1 hour. The number of each spectrum indicates the deposition temperature $(\mathrm{OC})$

\section{Supplementary Files}

This is a list of supplementary files associated with this preprint. Click to download.

- AuZrManuscriptSupplementaryInformation.pdf

- Figs1.pdf

- Figs2.pdf 